\title{
Medicação de alto risco: reflexão da ritalina
}

\author{
High risk medication: eflection of ritalin
}

Medicamentos de alto riesgo: reflexión de ritalin

João Pereira Filho ${ }^{1 *}$, Angelina Aryele Ramos Leal Rodrigues ${ }^{2}$, Marielly de Almeida Leão², Jaqueline Pereira de Sousa1, Juliana de Morais Araújo², Érica Gonçalves Lima ${ }^{3}$, Damião Dino da Costa ${ }^{4}$, Antonieldo Araújo de Freitas ${ }^{1}$, Maria Rivânia Cardoso ${ }^{1}$, Lorena Rocha Batista Carvalho .

\section{RESUMO}

Objetivo: Identificar os riscos e benefícios sobre a pratica de consumo não prescrito da Ritalina no Brasil. Métodos: Trata-se de uma Revisão Integrativa da Literatura, considerando artigos publicados nas bases de dados por meio dos descritores: Psicofármacos, Metilfenidato, Ritalina e Medicamentos, no período de 2014 a 2018. Resultados: Resultou-se em 5.224.529 publicações que após análise, chegou-se a 11 artigos que abordavam o tema. Resultou-se na construção de três categorias: experiência com o uso do medicamento, estratégias de obtenção do metilfenidato, potenciais riscos e benefícios do uso de metilfenidato. Considerações finais: Conclui-se que o consumo de metilfenidato apresenta diversos efeitos colaterais e adversos como: sonolência ou insônia, alucinações, problemas cardiovasculares, psicose, dependência e depressão, principalmente se for consumido de forma não prescrita. Portanto, entende-se que ainda há a necessidade de investir em programas de fiscalizações de medicações, assim como em programas de educação sobre os riscos e benefícios dos psicoestimulantes, evitando o processo medicalizante entre a indústria farmacêutica e a medicinam.

Palavras-chave: Psicofármacos, Metilfenidato, Ritalina, Medicamentos.

\section{ABSTRACT}

Objective: To identify the risks and benefits on the practice of non-prescribed consumption of Ritalin in Brazil. Methods: This is an Integrative Literature Review, considering articles published in the databases through the descriptors: Psychopharmaceuticals, Methylphenidate, Ritalin and Medicines, from 2014 to 2018. Results: There were 5,224,529 publications that after analysis, 11 articles were approached. It resulted in the construction of three categories: experience with the use of the drug, strategies for obtaining methylphenidate, potential risks and benefits of the use of methylphenidate. Final considerations: It is concluded that the consumption of methylphenidate has several side and adverse effects such as: drowsiness or insomnia, hallucinations, cardiovascular problems, psychosis, dependence and depression, especially if it is consumed in a non-prescribed manner. Therefore, it is understood that there is still a need to invest in medication inspection programs, as well as education programs on the risks and benefits of psychostimulants, avoiding the medicalizing process between the pharmaceutical industry and medication.

Keywords: Psychotropic drugs, Methylphenidate, Ritalin, Medicines.

\section{RESUMEN}

Objetivo: Identificar los riesgos y beneficios de la práctica del consumo no prescrito de Ritalin en Brasil. Métodos: Se trata de una Revisión de Literatura Integrativa, considerando artículos publicados en las bases de datos a través de los descriptores: Psicofármacos, Metilfenidato, Ritalina y Medicamentos, de 2014 a 2018. Resultados: Se registraron 5.224.529 publicaciones que después del análisis, se abordaron 11 artículos. El resultado fue la construcción de tres categorías: experiencia con el uso del fármaco, estrategias para la

\footnotetext{
1 Faculdade do Piauí (FAPI), Teresina - PI. * E-mail: enfjoaofilho@outlook.com

2 Faculdade Mauricio de Nassau (UNINASSAU), Teresina - PI.

3 Universidade Paulista (UNIP), Teresina - PI.

${ }^{4}$ Centro Universitário Santo Agostinho (UNIFSA), Teresina - PI.

${ }^{5}$ Universidade Brasil (UNIVBRASIL), Teresina - PI.
} 
obtención de metilfenidato, riesgos y beneficios potenciales del uso de metilfenidato. Consideraciones finales: Se concluye que el consumo de metilfenidato tiene varios efectos secundarios y adversos como: somnolencia o insomnio, alucinaciones, problemas cardiovasculares, psicosis, dependencia y depresión, especialmente si se consume de forma no prescrita. Por tanto, se entiende que aún existe la necesidad de invertir en programas de inspección de medicamentos, así como en programas de educación sobre los riesgos y beneficios de los psicoestimulantes, evitando el proceso de medicalización entre la industria farmacéutica y la medicación.

Palabras clave: Fármacos psicotrópicos, Metilfenidato, Ritalin, Medicamentos.

\section{INTRODUÇÃO}

Entre as Substâncias Psicoativas (SPAs) em uso terapêutico, destaca-se o metilfenidato (MTF), conhecido comercialmente no Brasil como Ritalina ou Concerta. Atualmente considerado um medicamento sob controle especial pela Portaria 344/984, incluso na lista de SPAs "A3", sujeita a notificação da receita "A", com estrutura química (C14H19NO2) semelhante à anfetamina. O MTF é uma substância indicada no tratamento do Transtorno de Déficit de Atenção e Hiperatividade (TDAH) e Narcolepsia, esta última, caracterizada como condição a qual se observa sonolência diurna excessiva em indivíduos (WILLE ARF e SALVI JO, 2018).

Somente nos anos 1950 que passou a ser comercializado, inicialmente na Suíça, logo após nos Estados Unidos da América (EUA) e por último na Alemanha e somente depois foi a ser vendido no Brasil. Liderando o ranking dos estimulantes mais vendidos no planeta, a Ritalina permanece com a sua produção em constante crescimento, o que demonstra a importância de uma fiscalização mais atuante por parte das autoridades competentes (MATOS HP et al. 2018).

Por ser uma medicação considerada de alto risco, o monitoramento da prescrição e do consumo contribui para um melhor desenvolvimento da vigilância sanitária evitando assim ocasionar dependência física, psíquica e outros tipos de riscos para a saúde (ANVISA, 2012).

Segundo pesquisa de Andrade LS (2018) e estudos da Associação Brasileira de Déficit de Atenção (ABDA), o TDAH acomete cerca de $3 \%$ a $5 \%$ da população brasileira, sendo mais frequente na população infantil do sexo masculino, o transtorno caracteriza-se pela desatenção, hiperatividade e impulsividade, dificultando o aprendizado do portador. No entanto, o transtorno é dividido em três subtipos, e é importante ressaltar que o indivíduo pode apresentar três diferentes graus de TDAH: leve, moderado ou grave.

A droga inicialmente é prescrita com indicação médica para pacientes adultos e crianças portadores de TDAH, porém, atualmente a Ritalina vem sendo muito utilizada de forma ilegal por jovens que não apresentam o transtorno, mas que procuram melhorar a capacidade de aprendizagem e evitando o cansaço e estresse mental, principalmente por estudantes que buscam alto desempenho intelectual, sendo conhecida hoje como "pílula da inteligência". Importante ressaltar que a maioria desses jovens não conhecem os efeitos colaterais que este medicamento proporciona, sendo que o consumo abusivo desta substância pode gerar graves consequências (ANDRADE LS, 2018; MELO RS, et al. 2020).

O fácil acesso a essa medicação faz com que esta preocupação cresça cada vez mais, tratando-se de uma anfetamina, apresenta um grande potencial químico, razão pela qual deve ser controlada e comprada com receita especial (CENTRO DE FARMACOVIGILÂNCIA, 2014). Alterações no sono, dependência, possíveis arritmias e em caso mais extremos podendo levar a suicídio, são consequências geradas por quem faz uso da Ritalina sem a devida prescrição (CENTRO DE FARMACOVIGILÂNCIA, 2014).

O objetivo deste estudo consistiu em identificar os riscos e benefícios sobre a prática de consumo não prescrito da Ritalina no Brasil.

\section{MÉTODOS}

O presente estudo trata-se de uma Revisão Integrativa da Literatura, referente à produção cientifica sobre a reflexão do uso de Ritalina no Brasil. Por se tratar de uma pesquisa de Revisão Integrativa da Literatura, o cenário de estudo compreendeu as bases de dados: LILACS, SciELO e MEDLINE. 
Desenvolveu-se a pesquisa de acordo com os propósitos da prática baseada em evidências, buscou-se sintetizar a realidade pesquisa com o objetivo de obter resultados referentes ao tema pesquisado (PRODANOV CC e FREITAS EC, 2013; MOREIRA MADM, et al., 2015).

Para o desenvolvimento desta revisão, seguiu-se um padrão em série de seis etapas com o intuito de manter um princípio metodológico, onde procedeu-se com: I- Identificação do tema a ser abordado; II- Coleta de dados na literatura; III- Emprego de critérios de inclusão e exclusão; IV- Elaboração e aplicação de um instrumento específico de coleta de dados; V- Análise crítica e Interpretação dos dados e finalmente VIApresentação dos resultados através de publicações (KUABARA CTM, et al., 2014; KINCHESCKI GF, ALVES R e FERNANDES TRT, 2015).

A população e amostra foi composta por artigos encontrados após criterioso refinamento utilizando os Descritores em Ciências da Saúde (DeCS), sendo eles: "psicofármacos", "metilfenidato", "ritalina" e "medicamentos", combinados com auxílio do Operador Booleano AND.

Utilizando critérios de inclusão selecionados especificamente para esta pesquisa, portanto determinou-se: trabalhos que estejam disponíveis nos bancos de dados online, em idioma português, com acesso gratuito, em formato de artigo científico e publicados nos últimos 5 anos (2014-2018) que retratem a temática em estudo.

Prosseguiu-se com aplicação de critérios de inclusão onde, determinou-se: trabalhos disponíveis na íntegra, em formato de artigo científico, com acesso gratuito, no idioma português, indexados nas referidas bases de dados citadas, publicados nos últimos 5 anos (2014-2018) e que retratassem a temática em estudo, restando 77 publicações com possibilidade de análise.

Foram excluídas publicações e produções não relacionadas à temática em estudo e assim como publicações de artigos repetidos, totalizando 11 artigos com o foco ao tema abordado. Com os resultados identificados após o processo de busca na íntegra, seguiu-se com a análise em três etapas. Inicialmente, realizado um estudo de cada publicação, permitindo-se a investigação e identificação de dados como: origem da base de dados indexada; ano de publicação; título do periódico; título do artigo; identificação dos autores; área de atuação dos integrantes; metodologia abordada; objetivo de estudo; amostra resultante; resultados e conclusões. Na segunda etapa, realizou-se uma análise interpretativa e síntese dos artigos de modo a captar a real ideia dos autores. Em uma última etapa foram apresentados os resultados através de uma análise dos artigos incluídos.

\section{RESULTADOS}

Prosseguiu-se a busca dos descritores isoladamente nas bases de dados sem a aplicação de nenhum filtro ou critério de inclusão (Tabela 1).

Tabela 1 - Distribuição de arquivos reunidos em cada base de acordo com os DeCS.

\begin{tabular}{cccc}
\hline DeCS & LILACS & MEDLINE & SciELO \\
\hline Psicofármacos & 978 & 19.385 & 271 \\
Metilfenidato & 141 & 6.457 & 107 \\
Ritalina & 6.453 & 93 & 16 \\
Medicamentos & 66.986 & 5.114 .931 & 8.711 \\
\hline Total & & & 5.224 .529
\end{tabular}

Fonte: PEREIRA FILHO J, et al., 2020.

Identificou-se através da busca dos descritores isoladamente 20.634 referências sobre "psicofármacos", 6.705 publicações sobre "metilfenidato", 6.562 referentes à "ritalina" e 5.190 .628 publicações sobre "medicamentos", totalizando 5.224 .529 referências publicadas.

Resultou-se em um grande número de publicações, prosseguimos em uma segunda etapa com o agrupamento aos pares dos descritores selecionados para este estudo, associados ao Operador lógico Booleano and, concomitantemente com os critérios de inclusão pré-estabelecidos (Tabela 2). 
Tabela 2 - Distribuição dos DeCS associados ao Operador Booleano and e critérios de inclusão.

\begin{tabular}{ccccc}
\hline DeCS + Operador Booleano and & LILACS & MEDLINE & SciELO & $\mathbf{N}^{\circ}$ \\
\hline Psicofármacos and metilfenidato & 3 & 0 & 0 & 3 \\
Psicofármacos and ritalina & 0 & 0 & 1 & 1 \\
Psicofármacos and medicamentos & 29 & 9 & 10 & 48 \\
Metilfenidato and ritalina & 6 & 1 & 4 & 11 \\
Metilfenidato and medicamentos & 5 & 1 & 3 & 9 \\
Ritalina and medicamentos & 3 & 1 & 1 & 5 \\
\hline Total & & & & $\mathbf{7 7}$ \\
\hline
\end{tabular}

Fonte: PEREIRA FILHO J, et al., 2020.

\section{DISCUSSÃO}

\section{O uso não prescrito da Ritalina}

O TDAH é um termo utilizado para denominar um diagnóstico psiquiátrico, ou transtorno do neurodesenvolvimento, considerado pela Organização Mundial da Saúde (OMS) como o psicoestimulante sintético mais vendido e consumido no mundialmente, chegando ao Brasil apenas em 1998 (DOMITROVIC N e CALIMAN LV, 2016; CRUZ AC, et al., 2016).

Habitualmente utilizado para classificar pessoas, principalmente crianças e jovens, que apresentam quadros, como características marcantes a distração, impulsividade e euforia/agitação psicomotora (CRUZ AC, et al., 2016). Na década de 50, era indicada para idoso no tratamento de distúrbios psicológicos diversos, como: cansaço, para os estados de psicose associada à depressão e narcolepsia, inércia e em episódios de confusão no envelhecimento (GONÇALVES CS e PEDRO MRM, 2018; ESHER A e COUTINHO T, 2017).

Estudos evidenciam que entre todas as Unidades Federativas (UF) do brasil, o Distrito Federal (DF) contabilizou o maior consumo de metilfenidato no triênio (2009, 2010 e 2011), sendo que a região Norte, na cidade de Rondônia obteve-se o maior consumo, enquanto que o Amazonas e Acre, tiveram o menor consumo registrado (ANVISA, 2012).

Evidencia-se pelos autores que o MTF é empregado para o tratamento de desordens psíquicas de crianças, adolescentes e comumente em idosos que apresentavam características diversas de psicose. Relatam ainda que primeiramente o MTF era aplicado na década de 50 para idosos que apresentavam algum tipo de alteração neuropsíquica, somente depois foi empregado para o tratamento de crianças e jovens.

Entende-se que o consumo da substância é alto, tornando o Brasil o segundo maior prescritor e consumidor de cloridrato de MTF para o tratamento, evidenciando assim a necessidade de fiscalização e regulamentação quanto a prescrição, compra e consumo (DOMITROVIC N e CALIMAN LV, 2016; CRUZ AC, et al., 2016).

Antigamente os transtornos mentais eram pouco diagnosticados em crianças, sendo consideradas desinquietas e travessas, e tão pouco recebiam o diagnóstico de TDAH. Já os adolescentes eram vistos como emocionalmente inconstantes e tímidos e esperava-se que quando chegassem a idade adulta seriam pessoas aptas para assumir suas responsabilidades (MARTINHAGO F, 2018).

No entanto, Moreira BMM, et al., (2017) evidencia que o TDAH é o distúrbio neuropsiquiátrico mais comum na infância, com prevalência de aproximadamente 5,0\% em crianças. Relata ainda que o tratamento proposto para esta idade é enquadrado em três tipos: terapia comportamental, farmacológico e a combinação das terapias farmacológica e comportamental.

Porém, os psicofármacos são atualmente prescritos como a principal e única alternativa terapêutica de tratamento para crianças com problemas educacionais, sociais, existenciais e emocionais (VIZOTTO LP e FERRAZZA DA, 2017). Estudos realizados no Brasil entre 2013 e 2014 pela Pesquisa Nacional sobre Acesso, Utilização e Promoção do Uso Racional de Medicamentos (PNAUM) evidência que o MTF é entre os medicamentos mais usados em doenças neuropsíquicas entre crianças de 6 a 12 anos (ESHER A e 
COUTINHO T, 2017). Assim, é evidente notar que de acordo com os resultados de alguns autores, o diagnóstico e TDAH e a prescrição de MTF era pouco utilizado antigamente para tratamento de desordens neuropsíquicas entre crianças e jovens.

No entanto, com o passar dos anos, o cloridrato de MTF foi utilizado como único e exclusivo componente para o tratamento de DTAH e Narcolepsia, deixando pouco ou não utilizado a terapia comportamental. Recentemente o diagnóstico adulto de TDAH tem sido alvo de muitos estudos, pois o seu tratamento medicamentoso e principalmente os efeitos que o uso da medicação veem proporcionando podem por vezes propiciar o uso inadequado com fins que não seja o do tratamento do TDAH (CALIMAN LV e RODRIGUES PHP, 2014).

Gonçalves CS e Pedro RMR (2018) referem que o consumo de MTF, veem sendo habitualmente utilizado a fim de obter um melhor aprimoramento cognitivo. Sabe-se que os estimulantes cerebrais são substâncias com capacidade de elevar o estado de alerta, além de possuírem propriedades antidepressivas, de melhora no humor e no desempenho cognitivo.

Dentre tais substâncias está MTF, cafeína, MDMA, bebidas energéticas, anfetaminas entre outras constituições, provocando no organismo consumidor a sensação de recompensa, euforia, atenção, motivação e excitação (MORGAN HL, et al., 2017). Apesar das propriedades produzidas no organismo por tais substâncias, observa-se que o risco de pendência química é elevado, e que por vezes podem ser prescritas e utilizadas para fins que não seja medicamentoso e sim para o aumento do desempenho cognitivo.

Relatos de estudos que muitos usuários de MTF vivenciam uma nova sensação, pois transformações cognitivas, de habilidades e do manuseio de tarefas rotineiras, passam a ser experimentadas com uma nova sensação após o início do uso da medição (CALIMAN VL e RODRIGUES HPH, 2014).

Esher A e Coutinho T (2017) referem que há usuários saudáveis que buscam ampliar sua capacidade cognitiva para prestar provas de concursos, produzir trabalhos que exijam muita concentração, aumentando sua produtividade e cumprindo com as suas responsabilidades e compromissos.

Entende-se que as sensações positivas que o cloridrato de MTF produz no corpo são vistas como boas e eficazes pelos usuários, há a necessidade de implementar uma política mais eficiente em busca de controlar a prescrição e expedição dos medicamentos, visto a possibilidade de dependência química e abuso de consumo da medicação seja um ponto negativo e que rodeiam os usuários de tal substância.

Portanto, a construção de protocolos para dispensação pública de MTF fazem parte de uma ação para tentar racionalizar os usos e minimizar empregos inadequados do medicamento (ESHER A e COUTINHO T, 2017). Apesar que os motivos justificados para o consumo de psicoestimulantes foram a compensação e privação de sono, melhorar raciocínio, atenção e/ou memória (MORGAN HL, et al., 2017).

\section{Estratégias de obtenção do metilfenidato}

$\mathrm{Na}$ cidade do Espírito Santo ocorrem a dispensação pública de medicamentos essenciais e excepcionais através das Farmácias Cidadãs, para um público reduzido a crianças, porém, com o passar dos anos o adulto diagnosticado, também passou a ter direito de solicitar gratuitamente o medicamento através das farmácias cidadãs (CALIMAN LV e RODRIGUES PHP, 2014).

Entende-se que o governo facilitou a aquisição de medicamentos de forma gratuita, através de programas como as Farmácias Cidadãs. Apesar de que há uma regra que apenas crianças e jovens possuíam o acesso aos medicamentos por tal farmácia, o adulto também passou a ter direito de adquirir de forma gratuita através do programa. O uso não terapêutico do MTF pode ser entendido como a busca dos indivíduos para superar seus limites ou simplesmente se adaptar à sociedade (MORGAN HL, et al., 2017). Este uso se faz, em grande maioria, por estudantes universitários, empresários e até por militares, como forma de otimizar a atenção, a produtividade e forma facilidade de enfrentar as tarefas diárias (COUTINHO T, et al., 2017). Em um estudo, realizado com universitários saudáveis que já haviam feito uso lícito ou ilícito de SPAs, evidenciam que os estudantes consumiam a substância para conseguir estudar por mais tempo, diminuindo o sono e reduzindo as sensações de tédios. 
Na mesma pesquisa os alunos relataram fazer o consumo não prescrito do MTF para potenciação cognitiva ou somente para fins recreativos. Sendo assim, os estudantes Medicina constituem um dos principais grupos vulneráveis ao consumo abusivo dessas substâncias com o objetivo de potencializar as atividades mentais, para fins recreativos, aumentar na aprendizagem, no estado de alerta e melhora da memória, contra episódios depressivos e elevação do desempenho acadêmico (MORGAN HL et al., 2017; MONTEIRO BMM et al., 2017). Apesar de existirem relatos de obtenção de estimulantes pela internet sem a devida prescrição médica, este ainda é o meio menos usado para tal fim.

A fonte mais comum de obtenção ilegal das SPAs é através de familiares, amigos ou parentes que adquirem a medicação por prescrição médica, mercado paralelo ou com médicos "enganados" por pacientes (MONTEIRO BMM, et al., 2017).

Neste mesmo pensamento de aquisição do medicamento de forma irregular, Esher A e Coutinho T (2017), relatam que em uma busca por Ritalina na rede social Facebook encontraram-se diversos grupos dedicados à discussão sobre o consumo de psicoestimulantes por quem tem TDAH, assim, como por concurseiros, além de grupos de venda sem receita.

No caso do TDAH, não existem exames laboratoriais que possam comprovar a patologia, sendo assim, o diagnóstico é apenas clínico. Portanto, qualquer pessoa pode facilmente forjar e receber o diagnóstico deste transtorno mental e facilmente obter uma prescrição para tratamento, bastando apresentar e comprovar as características que definem o transtorno. Portanto, o controle total sob essa prática profissional é inviável o que proporciona que a substância seja prescrita com uma finalidade e consumida por outra (MARTINHAGO F, 2018; ESHER A e COUTINHO T, 2017).

Assim, pessoas saudáveis podem forjar os sintomas do TDAH afim com conseguirem receitas do MTF, dificultando o controle das prescrições e compras do medicamento. Cabe então ao governo e demais setores responsáveis pela saúde medicamentosa da população a conscientização e educação em saúde, a fim de reduzir tal prática abusiva de consumo de SPAs, com fins de que não seja a do tratamento de alguma desordem mental.

\section{Potenciais riscos e benefícios do uso de metilfenidato}

O MTF é uma substância do grupo das anfetaminas que age como estimulante do sistema nervoso central e veem sendo comercializado como um "solucionador dos problemas de hiperatividade e da falta de atenção", em pacientes diagnosticados com TDAH (VIZOTTO LP e FERRAZZA DA, 2017). O MTF foi a substância cuja maior porcentagem de usuários relatou melhora no estado cognitivo.

Um fato importante observado é que age com efeito sobre a memória, raciocínio e concentração (MORGAN HL, et al., 2017). Segundo Gonçalves CS e Pedro RMR (2018) o principal problema não seria os riscos de efeitos colaterais, mas o potencial risco de uso abusivo desses medicamentos por seus usuários. O MTF é visto para a maioria dos usuários como uma solução para problemas de hiperatividade e falta de atenção, além disso, a geração médica já o traz como único meio de solucionar tal problema. Traz com características como o aumento da capacidade de resolver problemas do dia a dia, otimizando a atenção e produtividade.

Devido a tais características, o risco de seus usuários apresentarem ou desenvolverem dependência é elevado, apesar dos efeitos vistos como benéficos pelos seus consumidores, a Ritalina apresenta efeitos colaterais e adversos que por vezes podem ser maléficos.

Caliman LV e Rodrigues PHP (2014) relatam em seus estudos que a agressividade, alteração do humor, da produtividade, concentração, irritabilidade, foram reladas como sinais e sintomas evidenciados por adultos que fizerem o uso da medicação.

Gonçalves CS e Pedro MRM (2018) referem em suas pesquisas os efeitos que o medicamento teria, evidenciando o aprimoramento da performance cognitiva, destacando desde uma diminuição no sono que possibilitando o usuário estudar por mais tempo, aumento do foco, atuando no aumento da concentração e da vigília, no melhor rendimento e maior produtividade. No mesmo pensamento, são evidenciados através do 
uso da medicação por adultos o aumento do encorajamento para exercer funções cotidianas, aumento da produtividade no trabalho e da capacidade de questionar e argumentar (CALIMAN LV e RODRIGUES PHP, 2014).

Morgan HL, et al. (2017) destaca que o uso de estimulantes, trazem funções vistas como benéficas sobre funções mentais, como elevação do potencial desempenho acadêmico. Porém, foi demonstrado que o uso de estimulantes aumenta os níveis de estresse, reduzindo a qualidade de vida, tornando os usuários susceptíveis a doenças. Ressalta-se de que, mesmo com os efeitos vistos e relatados como positivos, é possível evidenciar reações e alterações maléficas que a substância provoca no organismo. Entende-se que a mesma, vai desde um simples aumento da capacidade cognitiva, até mesmo a doenças correlacionadas ao uso e abuso da Ritalina.

Os efeitos colaterais mais comuns do MTF quando usados por adultos são: insônia, enxaqueca, dor de estômago, tonturas, euforia, "olhar parado", desinteresse, irritabilidade, crises nervosas, hipertensão, dores de cabeça, alterações de apetite como a perda do apetite, enjoo, dor muscular e taquicardia (DOMITROVIC N e CALIMAN LV, 2016; MOREIRA MADM, et al., 2017; VIZOTTO LP e FERRAZZA DA, 2017).

Outro estudo apontou que o uso de estimulantes em adultos sem TDAH pode prejudicar o desempenho em tarefas que requerem adaptação, flexibilidade e planejamento. Apesar da Ritalina produzir um aumento da capacidade de produção e cognição nos adultos, é possível identificar o efeito inverso, prejudicando a capacidade de planejamento e de execuções de atividades diárias (MONTEIRO BMM, et al., 2017).

Vizotto LP e Ferrazza DA (2017) elencam em estudos consequências mais graves, pois o medicamento possui formação química similar à função da cocaína, provocando: tendência ao suicídio, comportamento agressivo, anorexia, alteração hepáticas, efeito cardiovasculares, alterações hormonais como possível diminuição do crescimento, ganho de peso, dependência química, entre outras graves condições.

Cruz AC, et al. (2016) e Martinhago F (2018) listam ainda alguns efeitos adversos como: alucinações, sonolência ou insônia, prejuízos na cognição, bem como efeitos no sistema cardiovascular, taquicardia, incluindo arritmia, hipertensão e parada cardíaca depressão, psicose, dependência e contrações musculares involuntárias.

Comparada à cocaína, o cloridrato de MTF pode provocar efeitos altamente graves em seus usuários, os autores expõem que mesmo que a Ritalina seja prescrita e vendida para pessoas com diagnósticos de TDAH, existem pessoas saudáveis, fazendo uso e principalmente o abuso da substância. Entende-se que assim como qualquer outro medicamento, existem os riscos e benefícios, os efeitos e reações, sendo assim, havendo o controle e regulação do uso da substância.

Percebe-se que há a necessidade de implantar e aderir para uma política e fiscalização de controle sobre a prescrição e uso do MTF, tanto para pessoas saudáveis, quanto para as que fazem uso com psicoestimulante, sem a necessidade de saúde e sim com fins de obter um maior desempenho durante as atividades do dia a dia.

\section{CONSIDERAÇÕES FINAIS}

Em relação ao uso prescrito do metilfenidato em crianças e adultos, existem problematizações a serem investigadas entre a indústria farmacêutica e a medicina, em relação ao contexto medicalizante. Portanto entende-se que há um discurso proposital por parte da indústria farmacêutica, que traz a noção de que o tratamento medicamentoso é a única e exclusiva solução. No que tange à experiência do uso do metilfenidato por pessoas saudáveis, ainda é elevado o número de usuários que fazem o uso da mediação, mesmo cintes dos efeitos e dos riscos correlacionadas ao uso e abuso da Ritalina. Entende-se que existe um úmero exorbitante de crianças diagnosticadas com déficit de atenção, porém, cabe ao profissional avaliar a real indicação da prescrição de cloridrato de metilfenidato. Evidenciou-se ainda que o uso não prescrito é rotineiro, assim como muitos usuários apresentam a capacidade de forjar os sintomas, a fim de serem diagnosticados com déficit de atenção e terem acesso a receitas. 


\section{REFERÊNCIAS}

1. ANDRADE LS, et al. Ritalina uma droga que ameaça a inteligência. Revista de Medicina e Saúde de Brasília, 2018; $7(1)$.

2. ANVISA. Boletim de Fármacoepidemiologia, SNGPC, ano 2, n. 2, jul. /dez., 2012.

3. CALIMAN LV, RODRIGUES PHP. A experiência do uso do metilfenidato em adultos diagnosticados com TDAH. Psicologia em Estudo, Maringá, 2014; 19(1): 125-134.

4. CENTRO DE FARMACOVIGILÂNCIA DA UNIFAL-MG N ${ }^{\circ} 26$. O uso da Ritalina para melhorar a concentração e raciocino de pessoas saudáveis, maio. 2014. Disponível em: https://www.unifalmg.edu.br/cefal/sites/default/files/Boletim_026_0.pdf. Acesso 05 abr 2019.

5. COUTINHO T, ESHER AF, OSARIO-DE-CASTRO CGS. Mapeando espaços virtuais de informação sobre TDAH e usos do metilfenidato. Physis Revista de Saúde Coletiva, Rio de Janeiro, 2017; 27(3): 749-769.

6. CRUZ AC, et al. Uma crítica à produção do TDAH e a administração de drogas para crianças. Estudos de Psicologia, 2016; 21(3): 282-292.

7. DORMITROVIC N, CALIMAN LV. As controvérsias sócio históricas das práticas farmacológicas com metilfenidato. Psicologia \& Sociedade. 2016; v. 29.

8. ESHER A, COUTINHO T. Uso racional de medicamentos, farmaceuticalização e usos do metilfenidato. Ciência \& Saúde Coletiva, 2017; 22(8): 2571-2580.

9. GONÇALVES CS, PEDRO RMR. Drogas da inteligência cartografando as controvérsias do consumo de ritalina para o aprimoramento cognitivo. Psicología, Conocimiento y Sociedad. Trabajos originales. 2018; 8(2): 71-94.

10. KINCHESCKI GF, ALVES R, FERNANDES TRT. Tipos de metodologias abordadas nas dissertações do programa de pós-graduação em administração universitária da Universidade Federal de Santa Catarina, no período de 2012 a 2014. V COLÓQUIO INTERNACIONAL DE GESTÃO UNIVERSITÁRIA - CIGU Desafios da Gestão Universitária no Século XXI. Mar del Plata - Argentina. ISBN: 978-85-68618-01-1. 16p. 2015.

11. KUABARA CTM, et al. Integração ensino e serviços de saúde: uma revisão integrativa da literatura. Rev. Min Enferm. 2014; 18(1): 195-201.

12. MARTINHAGO F, TDAH e Ritalina: neuro narrativas em uma comunidade virtual da Rede Social Facebook. Ciência \& Saúde Coletiva, 2018; 23(10): 3327-3336.

13. MATOS HP, et al. O uso da Ritalina em crianças com TDAH: uma revisão teórica, HumanÆE. Questões controversas do mundo contemporâneo. 2018; 12(2).

14. MELO RS, et al. RITALINA: consequências pelo uso abusivo e orientações de uso. Revista Científica Online. 2020; 12(1): ISSN 1980-6957.

15. MONTEIRO BMM, et al. Metilfenidato e melhoramento cognitivo em universitários: um estudo de revisão sistemática. SMAD, Rev. Eletrônica Saúde Mental Álcool Dog. 2017; 13(4): 232-242.

16. MOREIRA MADM, et al. Políticas públicas de humanização: revisão integrativa da literatura. Ciência \& Saúde Coletiva. $2015 ; 20(10): 3231-3242$.

17. MORGAN HL, et al. Consumo de Estimulantes Cerebrais por Estudantes de Medicina de uma Universidade do Extremo Sul do Brasil: Prevalência, Motivação e Efeitos Percebidos. Revista Brasileira de Educação Médica. 2017; 41(1): 102-109.

18. PRODANOV CC, FREITAS EC. Metodologia do trabalho científico [recurso eletrônico]: métodos e técnicas da pesquisa e do trabalho acadêmico. 2. ed. Novo Hamburgo: Feevale, 2013.

19. VIZOTTO LP, FERRAZZA DA. A infância na berlinda: Sobre rotulações diagnósticas e a banalização da prescrição de psicofármacos. Estudos de Psicologia. 2017; 22(2): 214-224.

20. WILLE ARF, SALVI JO. Prevalência do uso de Metilfenidato em acadêmicos de um centro universitário em Ji-Paraná - Rondônia, Brazilian Journal of Surgery and Clinical Research - BJSCR. 2018; 24(3): 13-19. 